\title{
A ROTARY FLOW CHANNEL FOR SHEAR STRESS SENSOR CALIBRATION
}

\author{
Allan J. Zuckerwar ${ }^{*}$ and Michael A. Scott ${ }^{\dagger}$ \\ NASA Langley Research Center, Hampton, Virginia, 23681
}

\begin{abstract}
A proposed shear sensor calibrator consists of a rotating wheel with the sensor mounted tangential to the rim and positioned in close proximity to the rim. The shear stress generated by the flow at the sensor position is simply $\tau_{w v}=\mu \mathrm{r} \omega / \mathrm{h}$, where $\mu$ is the viscosity of the ambient gas, $r$ the wheel radius, $\omega$ the angular velocity of the wheel, and $h$ the width of the gap between the wheel rim and the sensor. With numerical values of $\mu=31 \mu \mathrm{Pa}$ s (neon at room temperature), $r=0.5 \mathrm{~m}, \omega=754 \mathrm{~s}^{-1}(7200 \mathrm{rpm})$, and $h=50.8 \mu \mathrm{m}$, a shear stress of $\tau_{w}=231 \mathrm{~Pa}$ can be generated. An analysis based on one-dimensional flow, with the flow velocity having only an angular component as a function of the axial and radial coordinates, yields corrections to the above simple formula for the curvature of the wheel, flatness of the sensor, and finite width of the wheel. It is assumed that the sensor mount contains a trough (sidewalls) to render a velocity release boundary condition at the edges of the rim. The Taylor number under maximum flow conditions is found to be 62.3 , sufficiently low to obviate flow instability. The fact that the parameters entering into the evaluation of the shear stress can be measured to high accuracy with well-defined uncertainties makes the proposed calibrator suitable for a physical standard for shear stress calibration.
\end{abstract}

\section{Nomenclature}

$=$ radius of sensor

$=$ Fourier coefficient

$=$ area of sector of wheel

$=$ correction for radius of wheel

$=$ correction for finite width of wheel

$=$ correction for flatness of sensor

$=g(x)$ gap at location $x$

$=$ gap at location $x=0$

$=$ gap in region beyond sensor

$=$ wave number

$=$ modified Bessel Function, first kind

$=$ modified Bessel Function, second kind

$=$ Mach number

$=$ radius of wheel

$=$ radius of trough

$=$ Taylor number

$=$ flow velocity

$=r$-component of flow velocity

$=\theta$-component of flow velocity

$=z$-component of flow velocity

$=$ peripheral speed of wheel

$=$ width of wheel

* Senior Research Engineer, Advanced Model and Sensor Systems Branch, MS 2384 Langley Blvd, non-member AIAA.

${ }^{\dagger}$ Research Engineer, Advanced Sensing and Optical Measurement Branch, MS 49318 Langley Blvd., non-member AIAA. 


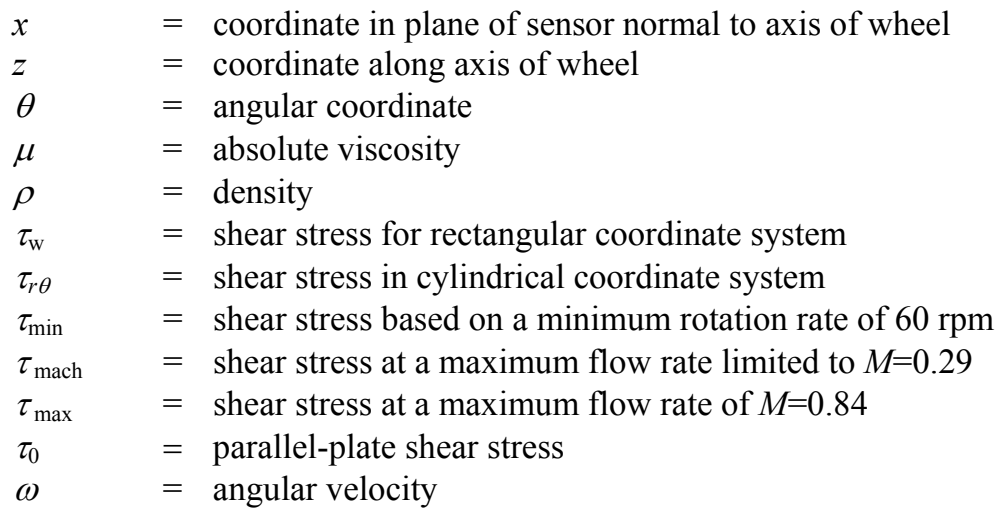

An overbar indicates a mean value.

\section{Introduction}

T $\mathrm{N}$ order to calibrate or characterize a shear stress sensor, the sensor must be exposed to a known shear stress. With the advent of micro-electrical mechanical systems (MEMS) shear stress sensors the size of new sensors is such that new calibration techniques become feasible. There are two methods commonly used to calibrate these sensors. The first is to infer the shear stress in-situ using some other measurement technique. This methodology typically involves the use of Preston or Stanton tubes and can only be done under certain limited conditions but is useful for fully developed turbulent boundary layer research. The other method used is to create a known flow thereby generating a known shear stress. To generate a known shear stress a precisely defined flow must be generated.

\section{A. Existing Methods for Generating a Known Shear Stress}

\section{Pressure Driven Flows}

One example of a pressure driven flow is a flow channel. A flow channel or laminar flow cell consists of two flat parallel plates of a finite width with the test gas, typically air or nitrogen, flowing between the plates and is one of the most commonly used methods for static shear stress calibrations. As long as the flow is laminar the velocity profile is well known and can be written in the following form:

$$
f(y)=U_{\infty}\left(1-\frac{y^{2}}{h^{2}}\right)
$$

where $f(\mathrm{y})$ is the velocity in the direction of the flow, $y$ is the normal distance from the centerline of the flow channel, $U_{\infty}$ is the centerline velocity, and $h$ is one-half the channel height. From this equation the shear stress in this type of channel is found to be (ignoring compressibility effects) and assuming adiabatic wall conditions:

$$
\tau_{w}=\frac{-2 \mu U_{\infty}}{h}
$$

where the negative sign is an artifact of the coordinate system. Additional corrections for compressibility have also been developed. This analysis only applies for static shear stress measurements. One disadvantage of this flow is that the velocity profile is parabolic while the laminar sub-layer has a linear velocity profile. This may not be an issue for sensors that directly detect the shear stress (e.g., floating element sensors) but any method that infers the shear stress (e.g., thermal sensors) must determine if there is sensitivity to the shape of the velocity profile.

\section{Displacement Driven Flows}

This category refers to plane-wave tubes with either a circular or rectangular cross section. These devices rely on Stokes layer excitation to generate a boundary layer that is dependent on the excitation frequency. This device is used for low level dynamic characterization. Versions of this concept have been reported in the literature for both 
zero mean flow and non-zero mean flow. Analysis of these devices is more complex than that of the simple analysis of a flow channel shown earlier and may be found in Ref.1 and Ref. 2.

\section{Boundary Driven Flows}

The proposed device is a new design for a boundary driven flow. A boundary driven flow is one where one or more of the boundaries moves in a continuous manner and the flow is generated by the no slip condition at the interface between the moving surface and the test media. One such device uses the face of a rotating wheel to generate a known flow with a linear velocity profile. This flow has components in the $\mathrm{r}, \theta$, and $\mathrm{z}$ direction. This is a complicated flow field even though it has a linear velocity profile.,

\section{B. Motivation for Development of a Rotary Flow Channel}

The primary motivation for designing a rotary flow channel is to enable precision calibration of MEMS shear stress sensors. Precision calibration requires a method for generating a known shear stress where the shear stress is dependent on a limited number of measurable or controllable quantities that can be determined with a known accuracy. The rotary flow channel has the potential to meet the requirements for a primary calibration standard for shear stress sensors. This device is intended to be one aspect of a comprehensive calibration scheme for MEMS shear stress sensors.

\section{Principle of Operation}

Figure 1 shows two parallel plates, the first moving at a constant speed $U$ with respect to the second. The velocity profile is linear between the plates. The shear stress $\tau_{\omega}$ on the surface of the stationary plate is found to be

$$
\tau_{w}=\mu \frac{\partial u}{\partial y}=\mu \frac{U}{g}
$$

where $\mu$ is the absolute viscosity of the medium, $u$ the $x$-component of velocity, $x$ the direction of motion, $y$ the normal direction, and $g$ the gap between the plates. Equation (1) suggests, in principle, an accurate method of generating a known shear stress for the calibration of skin-friction sensors, since the quantities on the right hand side can be determined very accurately. However, a calibrator based on rectilinear motion of continuous duration will prove impractical. (Even a device based on a tapedrive mechanism has severe practical limitations, especially regarding the stability of the tape motion). If the tape fabrication issues can be resolved we would recommend that a device based on this design be fully analyzed and considered for use as a device for the calibration of

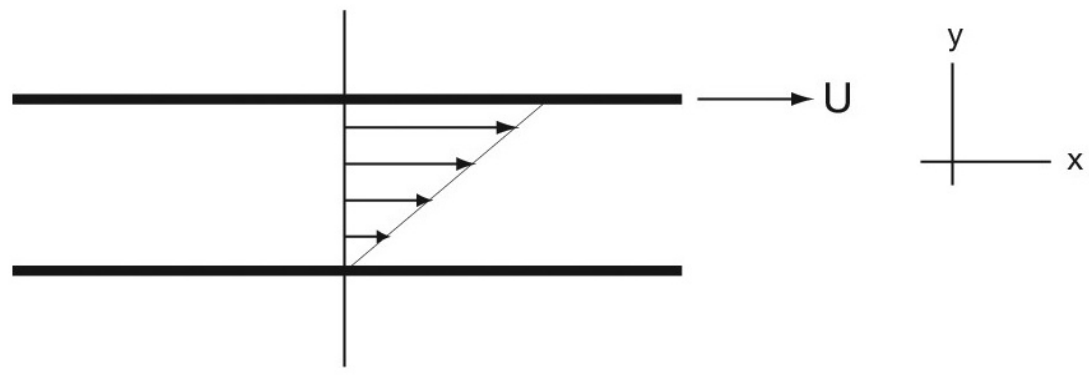
shear stress sensors.

Figure 1. Velocity profile between parallel plates, top plate moving and bottom plate stationary.

However, these considerations suggest that a more practical approach is to utilize a rotary motion for the generation of the flow needed to produce the shear stress.

Such a rotary flow channel is illustrated in Fig. 2. It consists of a wheel, sensor mount, trough, test medium, and supporting instrumentation. The wheel, having a radius $R_{1}$, rotates at a fixed angular velocity $\omega$, corresponding to a speed $U=R_{1} \omega$ at the surface of the wheel. The sensor mount, a flat plate containing the flush-mounted sensor to be calibrated, is positioned to realize a prescribed gap $g$. The trough has three functions: to entrain the ambient medium for flow into the gap, to suppress an axial component of the velocity, and to insure a velocity-release boundary condition at the edges of the wheel. Multiple configurations of the side wall design of the flow channel were considered and this version was selected for both ease of manufacturing as well as analytical simplicity. The 
supporting instrumentation monitors the wheel speed, gap, and temperature and pressure in the gap. The use of a

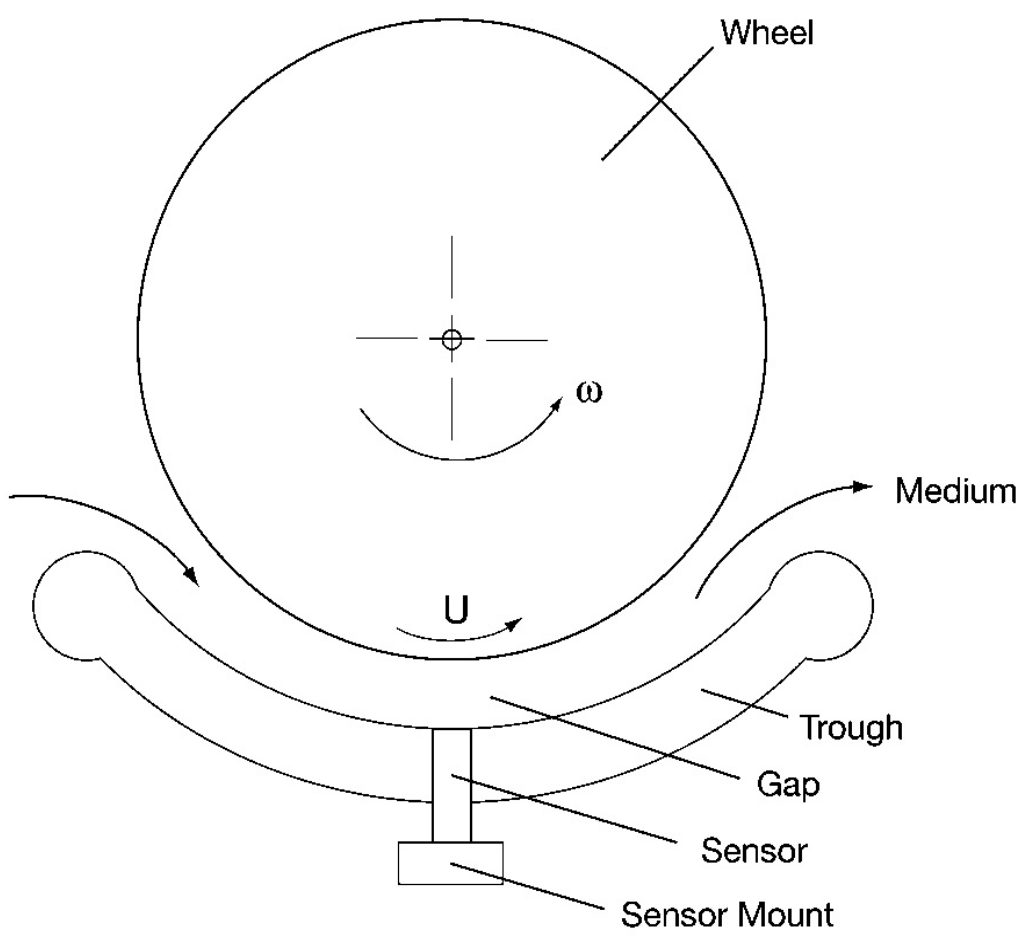

a)

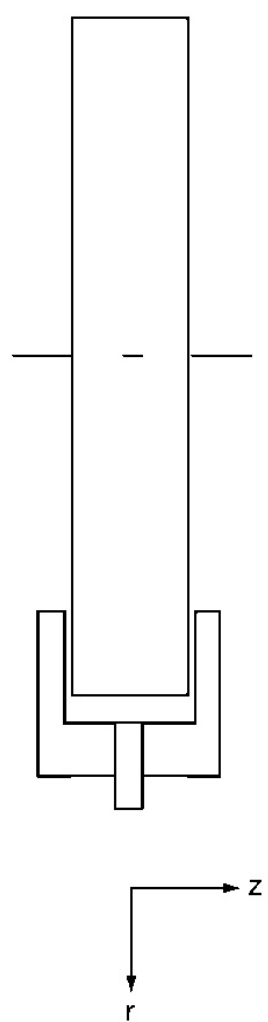

b)

Figure 2. Velocity profile between parallel plates, top plate moving and bottom plate stationary. Sidewalls of trough omitted from a) for clarity.

gas other than air, as well as safety considerations, dictates that the entire calibration assembly be situated in a robust, sealed test chamber.

Although the test medium can be air, the use of neon has several advantages. First, since it has the highest absolute viscosity of any common gas, a given shear stress can be generated at a lower wheel speed and larger gap than for air or other gases. Secondly, the high sound speed permits the generation of a given shear stress at a lower Mach number - an important consideration with regard to stability and compressibility effects. Thirdly, the high thermal conductivity facilitates the cooling of the medium and helps to maintain isothermal conditions in the gap. A comparison of pertinent physical properties between air and neon is shown in Table 1 .

Table 1: Properties of Air and Neon at $20^{\circ} \mathrm{C}$.

\begin{tabular}{|l|l|l|}
\hline & Air & Neon \\
\hline Absolute viscosity $\mu, \mathrm{Pa} \cdot \mathrm{s}$ & $1.83 \mathrm{E}-05$ & $3.11 \mathrm{E}-05$ \\
\hline Speed of sound $\mathrm{m} / \mathrm{s}$ & 343 & 449 \\
\hline Thermal conductivity, $\mathrm{W} /(\mathrm{m} \cdot \mathrm{K})$ & 56.2 & 107 \\
\hline Density $\rho$ at 1 atmosphere, $\mathrm{kg} / \mathrm{m}^{3}$ & 1.21 & 0.839 \\
\hline
\end{tabular}


The levels of attainable shear stress can be substantial. If we ignore the effects of compressibility and the possibility for violating the no slip boundary condition, consider a wheel having a radius $R_{1}=0.5 \mathrm{~m}$, rotating at $\omega=7200 \mathrm{rpm}$ (corresponding to $U=377 \mathrm{~m} / \mathrm{s}$ ) in neon, with a gap $g_{1}=76 \mu \mathrm{m}$. The shear stress generated at the surface of the trough is then $154 \mathrm{~Pa}$.

\section{Analysis}

\section{A. Shear Stress Without Corrections}

For a rotary flow channel, corrections to Eq. (3) must be made for the finite radius and width of the wheel and for the flatness of an inserted sensor. The first part of the analysis will consider a trough of circular contour; then a correction will be made for the flatness of the sensor.

Let the cylindrical coordinate system $(r, \theta, z)$ have its origin at the center of the wheel, as is shown in Fig. 3. We assume that the velocity components $u_{\theta}=u_{\theta}(r, z), u_{r}=u_{z}=0$. The boundary conditions are:
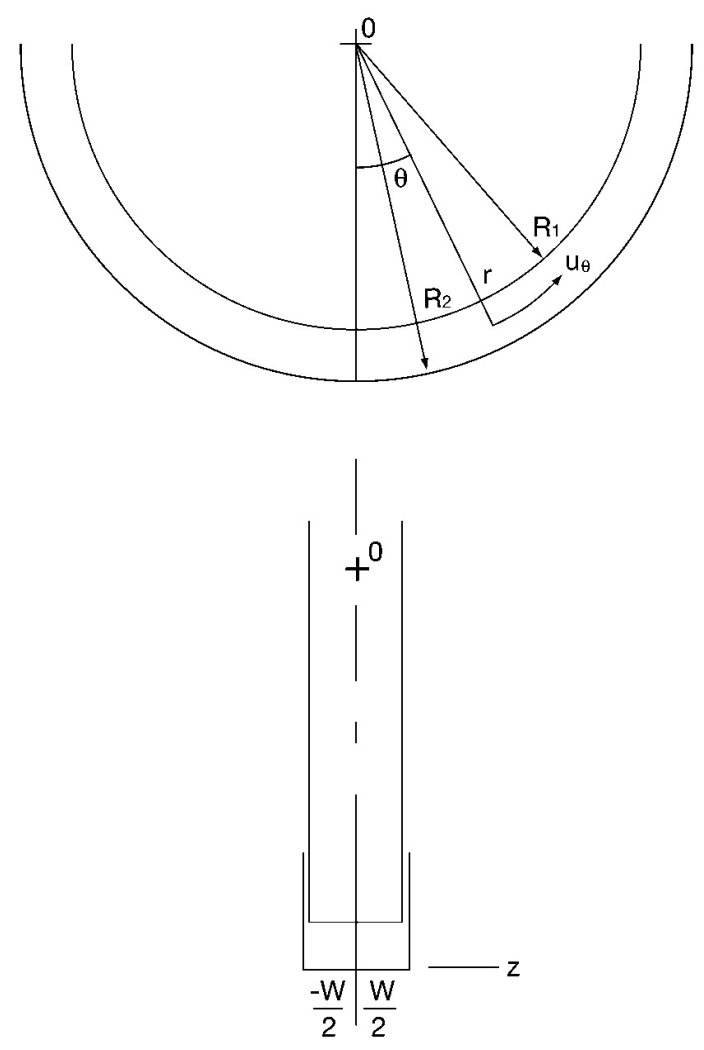

Figure 3. Cylindrical coordinate system for analysis of rotary flow channel showing both front and side views.

$$
\begin{gathered}
u_{\theta}\left(R_{1}, z\right)=U \\
u_{\theta}\left(R_{2}, z\right)=0 \\
u_{\theta}\left(r, \frac{-W}{2}\right)=u_{\theta}\left(r, \frac{W}{2}\right)=0,
\end{gathered}
$$

where $R_{2}$ is the radius of the trough and $W$ the width of the wheel. The Navier-Stokes Equations, continuity equation, and expression for the shear stress become: ${ }^{5}$

$$
\begin{gathered}
\nabla^{2} u_{\theta}=0 \\
\frac{\partial P}{\partial r}=\frac{\rho u_{\theta}^{2}}{r} \\
W \int_{g a p} u_{\theta} \mathrm{d} r=\text { constant }
\end{gathered}
$$




$$
\tau_{r \vartheta}=\mu\left(\frac{\partial u_{\theta}}{\partial r}-\frac{u_{\theta}}{r}\right)
$$

where $P$ is the pressure in the gap. The solution to Eq. (7a), satisfying the boundary conditions (6a-d), is the following (subscript for $k$ not shown for simplicity):

$$
u_{\theta}=U \sum_{n} a_{n}\left[\frac{-K_{1}\left(k R_{2}\right) I_{1}(k r)+I_{1}\left(k R_{2}\right) K_{1}(k r)}{-K_{1}\left(k R_{2}\right) I_{1}\left(k R_{1}\right)+I_{1}\left(k R_{2}\right) K_{1}\left(k R_{1}\right)}\right] \cos (k z)
$$

in which $k$ assumes the values

$$
k\left(=k_{n}\right)=\frac{n \pi}{W}(n=1,3,5, \ldots)
$$

to satisfy the boundary conditions (4c,d). In Eq. (6) $I_{1}$ and $K_{1}$ are modified Bessel functions of the first and second kinds.

Now, it will always be true that $k r \gg>1$ for all $r$ in the range $R_{1} \leq r \leq R_{2}$, in which case $I_{1}$ and $K_{1}$ approach their asymptotic limiting expressions:

$$
\begin{gathered}
I_{1}(k r) \rightarrow \frac{\exp (k r)}{\sqrt{2 \pi k r}} \\
K_{1}(k r) \rightarrow \frac{\exp (-k r)}{\sqrt{2 \pi k r}}
\end{gathered}
$$

both of which are also valid for $r=R_{1}$ and $R_{2}$. Upon substituting (8a,b) into (6), one can rewrite the latter as

$$
u_{\theta}=U \sum_{n} a_{n} \sqrt{\frac{R_{1}}{r}} \frac{\sinh \left[k\left(R_{2}-r\right)\right]}{\sinh \left[k\left(R_{2}-R_{1}\right)\right]} \cos (k z)
$$

Then, substituting Eq. (9) into (5d) yields for the shear stress anywhere in the gap

$$
\tau_{r \theta}=-\tau_{0} \sqrt{\frac{R_{1}}{r}} \sum_{n} a_{n}\left\{\frac{-k g_{1} \cosh \left[k\left(R_{2}-r\right)\right]-\frac{3}{2}\left(\frac{g_{1}}{r}\right) \sinh \left[k\left(R_{2}-r\right)\right]}{\sinh \left[k\left(R_{2}-R_{1}\right)\right]} \cos (k z)\right\}
$$

where $\tau_{0}$ is the stress in an equivalent flat-plate gap 


$$
\tau_{0}=\frac{\mu U}{g_{1}}
$$

and

$$
g_{1}=R_{2}-R_{1}
$$

is the gap prior to the insertion of the sensor to be calibrated. (The negative sign in Eq. (10) is related to the definition of the positive radial direction.) At the boundary $r=R_{1}$, the condition $u_{\theta}=U$ is realized if the Fourier coefficients in Eq. (9) are chosen as

$$
\begin{gathered}
a_{0}=\frac{1}{2} \\
a_{n}=(-1)^{\frac{n-1}{2}}\left(\frac{2}{\pi n}\right) \quad(n=1,3,5, \ldots)
\end{gathered}
$$

At the surface of the mounting plate $\left(r=R_{2}\right)$ the shear stress becomes

$$
\tau_{r \theta}=-\tau_{0} \sqrt{\frac{R_{1}}{R_{2}}}\left[\frac{1}{2}+\sum_{n}\left(\frac{2}{\pi}\right)(-1)^{\frac{n-1}{2}} \frac{k g_{1}}{\sinh \left(k g_{1}\right)} \frac{\cos (k z)}{n}\right](n=1,3,5, \ldots)
$$

The mean value of the shear stress across the width of the wheel becomes

$$
\bar{\tau}_{r \theta}=-\tau_{0} \sqrt{\frac{R_{1}}{R_{2}}}\left[\frac{1}{2}+\sum_{n}\left(\frac{2}{\pi}\right)^{2} \frac{k g_{1}}{\sinh \left(k g_{1}\right)} \frac{1}{n^{2}}\right](n=1,3,5, \ldots)
$$

The factors in Eq. (14) represent the flat parallel-plate shear stress, the correction due to the curvature of the wheel, and the correction for the finite width of the wheel, respectively. Upon insertion of the test sensor into the trough an additional correction is needed to account for the flatness of the sensor. Then the expression for the shear stress can be written

$$
\tau_{r \theta}=-\tau_{0}\left(1+C_{1}\right)\left(1+C_{2}\right)\left(1+C_{3}\right)
$$

where $C_{1}, C_{2}$, and $C_{3}$ are the three respective corrections.

\section{B. Correction for the Curvature of the Wheel}

The correction follows from Eq. (13): 


$$
C_{1}=\sqrt{\frac{R_{1}}{R_{2}}}-1 \approx-\frac{1}{2} \frac{g_{1}}{R_{1}}
$$

with the aid of Eq. (11b). Using values of the example at the end of Section II, the correction becomes

$C_{1}=-\frac{1}{2} \times \frac{76 \times 10^{-6}}{0.5}=-7.6 \times 10^{-5}$ or $-0.0076 \%$.

\section{Correction for the Finite Width of the Wheel}

The correction is attributable to the terms containing $\mathrm{kg}_{l}$ in Eq. (13). Without these terms the summation would have a value of $1 / 2$. Therefore

$$
C_{2}=\left(\frac{2}{\pi}\right)^{2} \sum_{n} \frac{k g_{1}}{\sinh \left(k g_{1}\right)} \frac{1}{n^{2}}-\frac{1}{2}(n=1,3,5, \ldots)
$$

The magnitude of the correction is shown as a function of $g_{1} / W$ in Fig. 4. If the wheel has a width of $0.0508 \mathrm{~m}$ and the gap remains $76 \mu \mathrm{m}$, then $g_{1} / W=0.0015$ and the correction amounts to $C_{2}=-6.6 \times 10^{-4}$ or $-0.066 \%$. To achieve a correction not exceeding $0.01 \%$ would require a ratio $g_{1} / W=0.0001$, corresponding to an impractical wheel width of $0.76 \mathrm{~m}$.

\section{Correction for Flatness of Test Sensor}

The sensor is mounted into a hole in the bottom surface of the trough with its rim flush at the intersection. Then the surface of the sensor will protrude into and thus narrow the gap, as illustrated in the front and top views shown in Fig. 5. However, there will be no surface discontinuities. The narrowing of the gap over the area of the sensor will lead to an increase in shear stress above that indicated in Eq. (11a). The correction will be based upon the mean value of the gap $g(x)$ over the surface of the sensor.

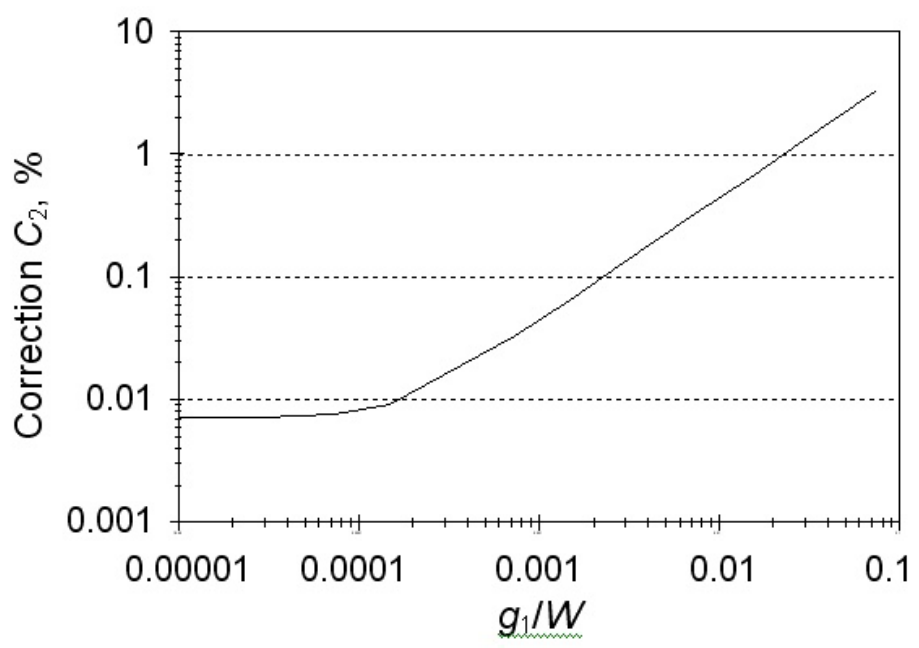

Figure 4. Magnitude of the correction $C_{2}$ due to the finite width of the wheel.

Let $g_{0}, g_{1}$, and $g(x)$ be the gap thicknesses at the center of the sensor, at the rim of the sensor, and at any distance $x$ from the center of the sensor, respectively. Geometrical considerations reveal that

$$
\left(R_{1}+g_{1}\right)^{2}-a^{2}=\left[R_{1}+g(x)\right]^{2}-x^{2}=\left(R_{1}+g_{0}\right)^{2}
$$



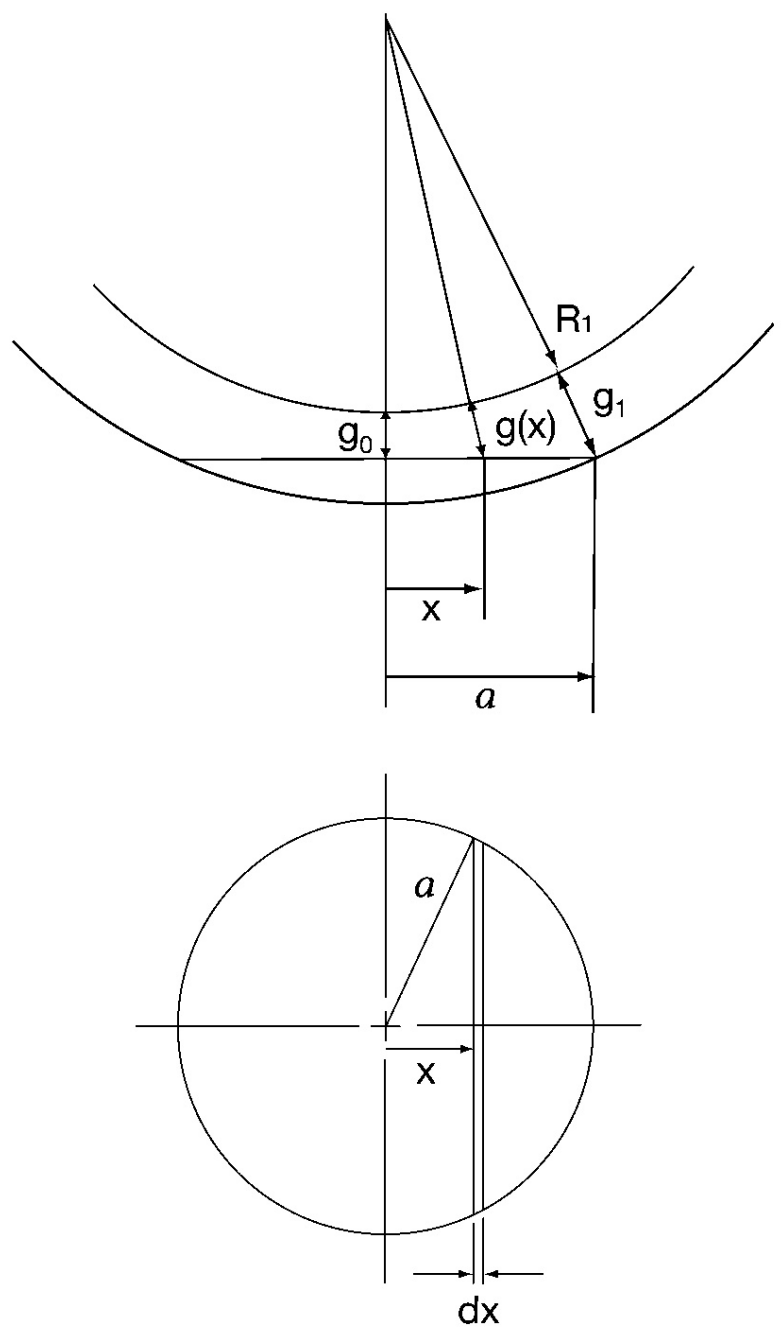

Figure 5. Detailed geometry of the sensor mount showing both front and top views.

from which the variable gap $g(x)$ is found:

$$
g(x)=\left(R_{2}^{2}-a^{2}+x^{2}\right)^{1 / 2}-R_{1}
$$

The elemental area $\mathrm{d} A$ about a chord at the distance $x$ from the center is

$$
\mathrm{d} A=2\left(a^{2}-x^{2}\right)^{1 / 2} \mathrm{~d} x
$$

Then the mean gap becomes 


$$
\bar{g}=\frac{1}{\pi a^{2}} \int_{-a}^{a} 2\left[\left(R_{2}^{2}-a^{2}+x^{2}\right)^{1 / 2}-R_{1}\right]\left(a^{2}-x^{2}\right)^{1 / 2} \mathrm{~d} x
$$

Under the assumption that $a / R_{2}<<1$, the integral can be evaluated in closed form, with the result:

$$
\bar{g}=\frac{2}{\pi a^{2}} \int_{-a}^{a}\left[g_{1}-\frac{1}{2} \frac{a^{2}-x^{2}}{R_{2}}\right]\left(a^{2}-x^{2}\right)^{1 / 2} \mathrm{~d} x=g_{1}\left(1-\frac{3}{8} \frac{a^{2}}{g_{1} R_{2}}\right)
$$

Upon inserting Eq. (21) into (11a), one finds the correction

$$
C_{3}=\frac{3}{8} \frac{a^{2}}{g_{1} R_{2}}
$$

Using the parameter values of the preceding examples, together with a sensor having a radius of $0.00127 \mathrm{~m}(0.05$ in.), one finds that the correction amounts to $C_{3}=\frac{3}{8} \times \frac{0.00127^{2}}{76 \times 10^{-6} \times 0.5}=0.016$ or $1.6 \%$. This correction, in contrast to the others, leads to an increase in the shear stress.

The small constriction in the flow channel caused by the presence of the sensor causes an acceleration of the flow into the gap. If the flow remains incompressible, then the invariance of the volume velocity causes Eq. (5c) to take the following form:

$$
\overline{u_{\theta}}=\frac{U g_{1}}{2 g}
$$

the effect of which is accounted for in the correction $C_{3}$. Unfortunately $\mathrm{C}_{3}$ also shows a limiting factor for this design. As the radius of the sensor increases the correction increases by the square. For the design example if we limit $C_{3}$ to 0.1 that imposes a limit on the sensor radius of $0.0032 \mathrm{~m}(0.13 \mathrm{in}$.). If a larger gap size can be used to generate the needed shear stress then the size of the sensor may increase and maintain the value of $\mathrm{C}_{3}$.

\section{E. Stability of the Flow}

The rotary flow channel represents a special case of Couette flow, which displays a multitude of instability modes even at low Reynolds numbers. The criterion for the onset of instability is based on the Taylor number ${ }^{6}$ :

$$
T a=\frac{2 \rho^{2} \omega^{2} R_{1} g_{1}^{3}}{\mu^{2}}
$$

A Taylor number not exceeding the critical value of 1708 is said to insure a stable flow. Upon inserting above parameter values and those found in Table I, one finds the Taylor number (in neon): 


$$
T a=\frac{2 \times 0.839^{2} \times 754^{2} \times 0.5 \times\left(76 \times 10^{-6}\right)^{3}}{\left(3.11 \times 10^{-5}\right)^{2}}=181
$$

Since the Taylor number is far below critical, the rotary channel flow is expected to be stable.

\section{F. Centrifugal Pressure on the Sensor}

The centrifugal pressure on the sensor follows directly from Eq. (5b):

$$
P=\rho \int_{R_{2}}^{R_{1}} \frac{u_{\theta}^{2} \mathrm{~d} r}{r}
$$

Upon inserting the value of $u_{\theta}$ in the limit of small $\mathrm{kg}_{I}$

$$
u_{\theta} \approx \frac{U\left(R_{2}-r\right)}{g_{1}}
$$

one finds

$$
P\left(R_{2}\right)=-\rho\left(\frac{U}{g_{1}}\right)^{2}\left[R_{2}^{2} \ln \left(\frac{R_{2}}{R_{1}}\right)+\frac{g_{1}}{2}\left(R_{1}-3 R_{2}\right)\right]
$$

With the parameter values used above, the centrifugal pressure exerted on the sensor amounts to $2.35 \mathrm{~atm}$. This will not load the sensor if the sensing element is back vented. Further, the Knudsen number, defined here as the mean free path over the thickness of the gap, is $9.4 \times 10^{-8} / 76 \times 10^{-6}=0.0012$, indicating that the flow is well within the domain of continuum theory.

\section{IV. Design Review and Possible Extensions}

By choosing a single value for the radius of the wheel, $R_{1}$, we obtain a simple correlation between the intended gap $\left(g_{1}\right)$, peripheral speed of the wheel $(U)$, and the generated shear stress as shown in Eq. 3. Of more interest is a design guideline for range of shear stress in air and neon for different gap sizes. Table 2 and Table 3 show this

Table 2: Achievable shear stress versus gap size in Table 3: Achievable shear stress versus gap size in air at $20^{\circ} \mathrm{C}$ for $R_{1}=0.5 \mathrm{~m}$ and minimum $\omega=60 \mathrm{rpm}$, neon at $20^{\circ} \mathrm{C}$ for $R_{1}=0.5 \mathrm{~m}$ and minimum $\omega=60 \mathrm{rpm}$, the mach number limited shear stress at $\omega=1899.74$ the mach number limited shear stress at $\omega=\mathbf{2 4 8 6 . 8 3}$ rpm, and maximum shear stress at $\omega=5500 \mathrm{rpm}$. rpm, and maximum shear stress at $\omega=7200 \mathrm{rpm}$.

\begin{tabular}{|l|l|l|l|}
\hline Gap, $\boldsymbol{\mu m}$ & $\tau_{\text {min }}$, Pa & $\tau_{\text {mach }}$, Pa & $\tau_{\text {max }}$, Pa \\
\hline 400 & 0.14 & 4.55 & 13.18 \\
\hline 300 & 0.19 & 6.07 & 17.57 \\
\hline 200 & 0.29 & 9.10 & 26.35 \\
\hline 150 & 0.38 & 12.14 & 35.13 \\
\hline 100 & 0.57 & 18.20 & 52.70 \\
\hline 75 & 0.77 & 24.27 & 70.27 \\
\hline 50 & 1.15 & 36.41 & 105.4 \\
\hline
\end{tabular}

\begin{tabular}{|l|l|l|l|}
\hline Gap, $\mu \mathrm{m}$ & $\tau_{\min }$, Pa & $\tau_{\text {mach }}$, Pa & $\tau_{\max }$, Pa \\
\hline 400 & 0.24 & 10.12 & 29.31 \\
\hline 300 & 0.33 & 13.50 & 39.08 \\
\hline 200 & 0.49 & 20.25 & 58.62 \\
\hline 150 & 0.65 & 27.00 & 78.16 \\
\hline 100 & 0.98 & 40.50 & 117.24 \\
\hline 75 & 1.30 & 53.99 & 156.33 \\
\hline 50 & 1.95 & 80.99 & 234.49 \\
\hline
\end{tabular}


information for air and neon respectively. The minimum shear stress is based on $\omega=60 \mathrm{rpm}$ and scales linearly for decreased values of $\omega$. The shear stress listed for $\tau_{\text {mach }}$ corresponds with a linear velocity limited to $M=0.29$. The maximum shear stress corresponds to a linear velocity where $M=0.84$.

These tables are useful for quickly determining the gap size needed to generate the shear stress range of interest. Comparing these values with the shear stress obtainable using a flow channel shows that the flow channel generates a higher shear stress for a given height. One advantage with the rotary flow channel is while it needs to be completely enclosed, there is no exhaust to the ambient environment required. This simplifies the use of neon for the higher viscosity to obtain higher shear stress.

The rotary flow channel, as well as the tape based design; both have the potential to generate a small dynamic shear stress about a mean value. This dynamic component while small compared to the maximum shear stress attainable would be achievable with a wide range of mean values at a range of frequencies dependent on the details of implementation. The method to do this would be to very precisely move or vibrate the trough at a known magnitude and frequency. The analysis of this flow would be more complex than the analysis shown here since the gap would not be constant except at the initial position of the trough as analyzed previously.

\section{Conclusions}

The rotary flow channel has many desirable characteristics for generating a known shear stress with the potential for providing a dynamic shear stress capability as well. The corrections needed for a practical sized device are small and easily determined. To keep the corrections small does impose a very stringent requirement on the sensor size but for MEMS devices this should not be insurmountable and may even be advantageous in that a flush mounted device may be able to be pre-installed in a plug type test article that is then mounted with only a portion of the sensor die exposed to the calibration flow. Additional analysis needs to be done with particular emphasis on wall slip conditions. Since this is not a pressure driven flow compressibility should not be an issue with the possible exception of the region at the sensor installation due to the non-uniformity in the gap in this region.

\section{References}

${ }^{1}$ Sheplak, M., Padmanabhan, A., Schmidt, M. A., and Breuer, K. S., "Dynamic Calibration of a shear stress sensor using Stokes layer excitation," 1998, AIAA Paper 1998-585.

${ }^{2}$ Chandrasekaran, V., Cain, A., Nishida, T., and Sheplak, M., "Dynamic calibration technique for thermal shear stress sensors with variable mean flow," $38^{\text {th }}$ Aerospace Sciences Meeting and Exhibit, January 10-13, 2000, AIAA Paper 2000-0508.

${ }^{3}$ Yongxiang, L., and Naguib, A. M., "A High-Frequency Oscillating-Hot-Wire Sensor for Near-Wall Diagnostics in Separated Flows," $42^{\text {nd }}$ Aerospace Sciences Meeting and Exhibit. January 5-8, 2004, AIAA Paper 2004-1041.

${ }^{4}$ Khoo, B. C., Chew, Y. T., and Lim, C. P. "The Flow Between a Rotating and a Stationary Disc: Application to Near-Wall Hot-Wire Calibration," Measurement Science Technology 9: 1988, 650-658.

${ }^{5}$ Schlichting, H., Boundary-Layer Theory, Springer-Verlag, New York, 1987, Chap. 1.

${ }^{6}$ Chossat, P., and Iooss, G., The Couette-Taylor Problem, Springer-Verlag, New York, 1991, Chap. 1. 